a 
Ph. Blanchard und E. Brüning

\section{Distributionen und Hilbertraumoperatoren}

Mathematische Methoden der Physik 
Prof. Dr. Philippe Blanchard

Fakultät für Physik

Universität Bielefeld

D-33501 Bielefeld

Bundesrepublik Deutschland

Prof. Dr. Erwin Brüning

Department of Mathematics

University of Cape Town

Rondebosch 7700

South Africa

Das Werk ist urheberrechtlich geschützt.

Die dadurch begründeten Rechte, insbesondere die der Übersetzung, des Nachdruckes, der Entnahme von Abbildungen, der Funksendung, der Wiedergabe auf photomechanischem oder ähnlichem Wege und der Speicherung in Datenverarbeitungsanlagen, bleiben, auch bei nur auszugsweiser Verwertung, vorbehalten.

(C) 1993 Springer-Verlag Wien

Ursprünglich erschienen bei Springer-Verlag/Wien 1993

Satz: Reproduktionsfertige Vorlagen der Autoren

Gedruckt auf säurefreiem, chlorfrei gebleichtem Papier - TCF 
Res Jost (10.I.1918 - 3.X.1990) und Jurko Vladimir Glaser (21.IV.1924 - 22.I.1984) in Erinnerung gewidmet, die für uns beide verehrte Vorbilder und für einen von uns (Ph.B.) auch liebe Freunde waren. 


\section{Vorwort}

Vorlesungen der modernen Theoretischen Physik setzen unter anderem Grundkenntnisse in der Theorie der verallgemeinerten Funktionen (insbesondere Distributionen) und in der Theorie der linearen Operatoren auf Hilbert-Räumen voraus. Diesem Umstand wurde im Lehrplan der Fakultät für Physik der Universität Bielefeld Rechnung getragen und ein Kurs "Mathematische Methoden der Physik" eingerichtet, der von den Studenten zum Vordiplom absolviert werden muß. Dementsprechend wird dieser einsemestrige Kurs seit vielen Jahren regelmäßig abgehalten. Dieses Buch entstand aus den Vorlesungen, die die Autoren im Wechsel und unter inhaltlicher Absprache über einen Zeitraum von fast zehn Jahren gehalten haben. Als Hauptziel dieses Kurses kristallisierte sich bald heraus, mathematische Grundkenntnisse zu vermitteln, wie sie vor allem in der Quantenmechanik und in der relativistischen Quantenfeldtheorie, aber nicht nur dort, benötigt werden. Die zahlreichen Wiederholungen dieser Vorlesungen erlaubten es, viele Variationen im Inhalt und in der Darstellungsform des Stoffes zu testen. Das Buch ist daher durchaus umfangreicher als die einzelnen Vorlesungen geraten, hinzugekommen sind vor allem detailliertere mathematische Beweise und zahlreiche Beispiele und Hinweise auf mögliche Anwendungen. Wie schon in der Vorlesung selbst haben wir uns um Motivation der eingeführten mathematischen Begriffe und Methoden durch Problemstellungen der Physik bemüht.

Dementsprechend richtet sich dieses Buch an Studenten der Physik, die Interesse an einer genaueren mathematischen und begrifflichen Erfassung physikalischer Problemstellungen haben und an Studenten der Mathematik, die Physik als Quelle mathematischer Problemstellungen und als Anwendungsgebiet mathematischer Theorien kennenlernen möchten.

Es wird vorausgesetzt, daß der Leser etwa den Stoff eines dreisemestrigen Kurses "Differential- und Integralrechnung" und eines zweisemestrigen Kurses "Lineare Algebra" beherrscht. Auf dieser Basis beginnt unser Buch im ersten Teil mit einer Einführung in die Theorie der Distributionen, die auch einige Grundbegriffe der linearen Funktionalanalysis vermittelt. Der zweite Teil baut darauf auf und gibt eine auf das Wesentliche beschränkte Einführung in die Theorie der linearen Operatoren in Hilbert-Räumen. Beide Teile werden von je einer Übersicht begleitet, die die zentralen Ideen und Begriffe knapp erläutert und den inhaltlichen Kern dieses Teils beschreiben. Einige Anhänge stellen in elementarer Weise einige grundlegende Konstruktionen und Konzepte der Funktionalanalysis dar und entwickeln einige wichtige Konsequenzen.

Aus verschiedenen Gründen hat sich die Herstellung dieses Buches erheblich verzögert. In der ersten Phase hat Frau R. Pludra durch ihren Einsatz beim Schreiben des Manuskriptes wesentlich zum Herstellungsprozess beigetragen. Die Erstellung der TEX-Version des Manuskriptes in der zweiten Phase verdanken wir dem unermüdlichen Einsatz von Frau G. Eickmeyer und Frau H. Litschewsky. Allen gilt unser aufrichtiger Dank. Unsere Kollegen G. Bolz, Th. Ellerbrock, V. Enß, S. Golin, T. Griese, C. Hellmich, M. Lefeld, J. Loviscach, Ch. Menzel, C. Neumann, U. Oppitz, J. Potthoff, G. Sommer, O. Steinmann, L. Streit und J. Stubbe haben uns durch Kommentare, Verbesserungsvorschläge und Skriptkorrekturen direkt oder indirekt geholfen. Dafür möchten wir uns herzlichst bedanken.

Enger und Durban, 1993

Ph. Blanchard

E. Brüning 


\section{Inhaltsverzeichnis}

Abkürzungen $\quad$ xi

Teil I: Einführung in die Distributionentheorie

Übersicht 1

0 Einleitung 2

1 Testfunktionenräume $\quad 10$

2 Distributionen $\quad 27$

3 Elementare Rechenoperationen für Distributionen 36

4 Darstellungssatz für Distributionen - Radon Maße $\quad 58$

5 Tensorprodukt und Faltung $\quad 63$

6 Anwendungen der Faltung 83

7 Holomorphe Funktionen $\quad 102$

8 Fourier-Transformation $\quad 112$

Teil II. Einführung in die Theorie der Hilbert-Räume

Übersicht 145

9 Einleitung $\quad 151$

10 Prä-Hilbert-Raum und Hilbert-Raum 155

11 Geometrie eines Hilbert-Raumes $\quad 170$

12 Separable Hilbert-Räume 181

13 Direkte Summen und Tensorprodukte von Hilbert-Räumen 194

14 Topologische Probleme in Hilbert-Räumen: Kompaktheit und schwache Topologie 199

15 Lineare Operatoren in Hilbert-Räumen 210

16 Quadratische Formen 228

17 Beschränkte lineare Operatoren in Hilbert-Räumen 237

18 Spezielle Klassen beschränkter linearer Operatoren $\quad 252$

19 Operatoren der Quantenmechanik $\quad 271$

20 Spektraltheorie für lineare Operatoren im Hilbert-Raum $\quad 279$

21 Der Spektralsatz für selbstadjungierte Operatoren 290

22 Interpretation des Spektrums eines selbstadjungierten Operators 329

\section{Anhänge}

A1. Vervollständigung eines metrischen Raumes $\quad 349$

A2. Metrisierbare HLKTVR's $\quad 352$

A3. Der Satz von Baire. Bairesche Räume $\quad 354$

A4. Anwendungen des Satzes von Baire $\quad 357$

A5. Bilineare Funktionale $\quad 361$

A6. Beweis von Satz 5.1 und Satz 5.2 a 363

$\begin{array}{ll}\text { Literatur } & 367\end{array}$

$\begin{array}{ll}\text { Sachverzeichnis } & 369\end{array}$ 


\section{Abkürzungen}

$\mathbf{R}$

C

$\mathbf{K}$

$\mathbf{K}^{n}$

$\mathbf{R}_{+}$

$A \pm B$

$\Lambda M$

$A \backslash B$

$\mathcal{C}(\Omega)=C(\Omega ; \mathbf{K})$

supp $f$

$\mathcal{C}_{o}(\Omega)$

$\mathcal{C}^{k}(\Omega)$

$D^{\alpha}=\frac{\partial^{|\alpha|}}{\partial x_{1}^{\alpha_{1}} \cdots \partial x_{n}^{\alpha_{n}}}$

$\mathcal{D}_{K}(\Omega)$

$\mathcal{D}(\Omega)$

$\mathcal{S}(\Omega)$

$\mathcal{E}(\Omega)$

LKTVR

HLKTVR

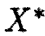

Körper der reellen Zahlen

Körper der komplexen Zahlen

Körper der reellen oder komplexen Zahlen

$\mathbf{K}$-Vektorraum der $n$-Tupel von Zahlen in $\mathbf{K}$

$[0, \infty]$ nichtnegative reelle Zahlen

$\{a \pm b \mid a \epsilon A, b \epsilon B\}$ für Teilmengen $A$ und $B$ eines Vektorraumes $V$

$\{\lambda \cdot u \mid \lambda \epsilon \Lambda, u \epsilon M\}$ für eine Teilmenge $\Lambda \subset \mathbf{K}$ und eine Teilmenge $M$ eines Vektorraumes über $\mathbf{K}$.

Differenzmenge $=$ Menge aller Punkte in $A$, die nicht zu $B$ gehören

Vektorraum aller stetigen Funktionen

$f: \Omega \rightarrow \mathbf{K}$ für eine offene Menge $\Omega \subseteq \mathbf{R}^{n}$

Träger der Funktion $f$

Vektorraum aller stetigen Funktionen $f: \Omega \rightarrow \mathbf{K}$ mit kompaktem Träger supp $f \subset \Omega \subseteq \mathbf{R}^{n}$

Vektorraum aller Funktionen, die stetige Ableitungen bis zur Ordnung $k=0,1,2, \ldots$ besitzen

Ableitungsmonom der Ordnung $|\alpha|=\alpha_{1}+\cdots+\alpha_{n}$ auf $\mathcal{C}^{k}(\Omega),|\alpha| \leq k$, für $\Omega \subset \mathbf{R}^{n}$ offen

Vektorraum aller beliebig oft differenzierbaren

Funktionen $f: \Omega \rightarrow \mathbf{K}$, deren Träger supp $f$ in der kompakten

Teilmenge $K$ von $\Omega \subset \mathbf{R}^{n}$ enthalten ist; versehen mit der

Topologie der gleichmäßigen Konvergenz für alle Ableitungen

induktiver Limes der Räume $\mathcal{D}_{K}(\Omega), K \subset \Omega$ kompakt;

Testfunktionenraum aller $\mathcal{C}^{\infty}$-Funktionen $f: \Omega \rightarrow \mathbf{K}$ mit

kompaktem Träger in der offenen Menge $\Omega \subseteq \mathbf{R}^{n}$

Testfunktionenraum aller $\mathcal{C}^{\infty}$-Funktionen $f: \Omega \rightarrow \mathbf{K}$, die mit allen Ableitungen überpolynomial abfallen

Testfunktionenraum aller $\mathcal{C}^{\infty}$. Funktionen $f: \Omega \rightarrow \mathbf{K}$

lokalkonvexer topologischer Vektorraum

Hausdorffscher lokalkonvexer topologischer Vektorraum

algebraischer Dualraum eines Vektorraumes $X$ 


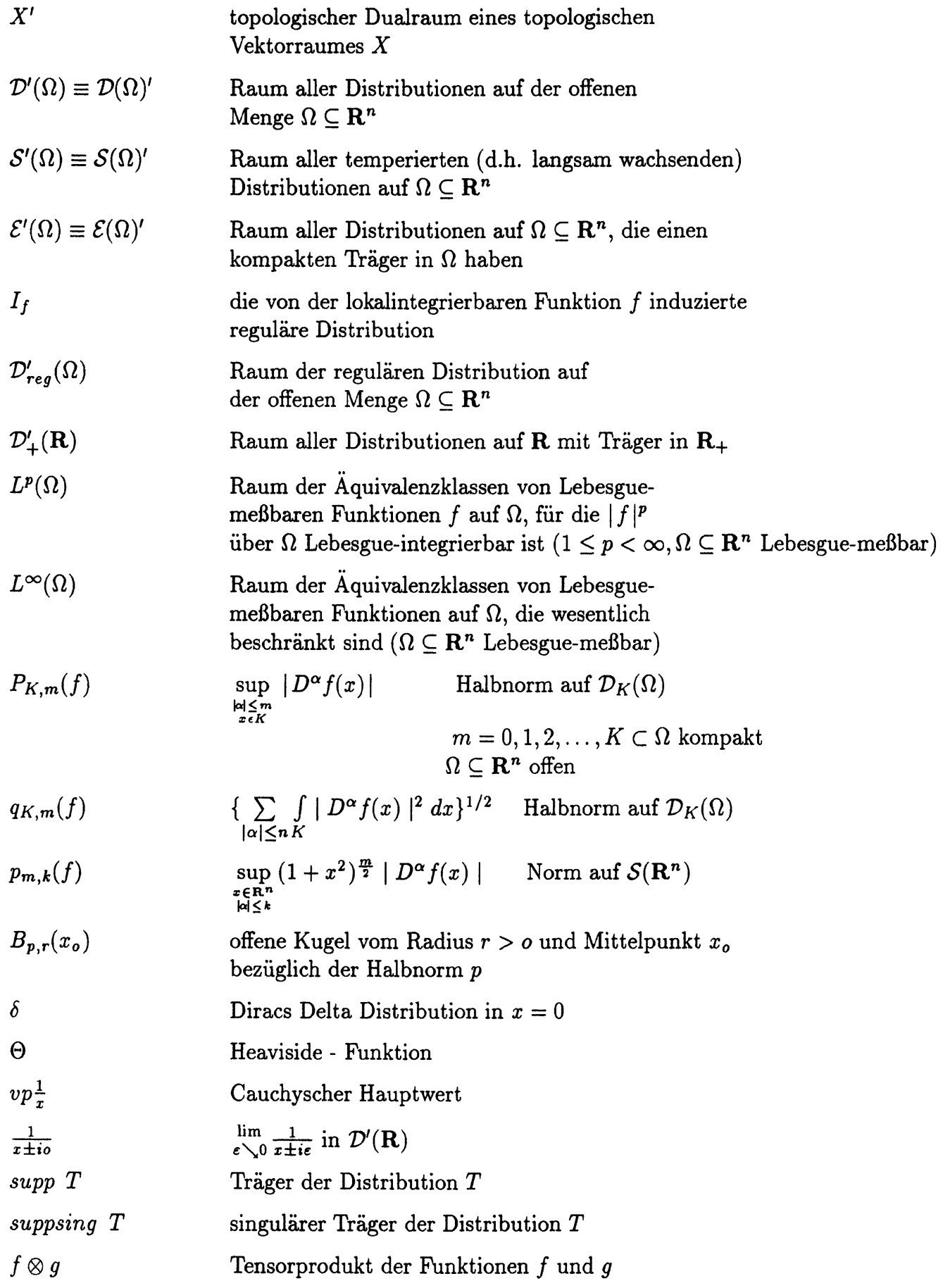

Diracs Delta Distribution in $x=0$

Heaviside - Funktion

Cauchyscher Hauptwert

$\lim _{\varepsilon \searrow 0} \frac{1}{x \pm i \varepsilon}$ in $\mathcal{D}^{\prime}(\mathbf{R})$

Träger der Distribution $T$

singulärer Träger der Distribution $T$

Tensorprodukt der Funktionen $f$ und $g$ 
$T \otimes S$

$\mathcal{D}\left(\mathbf{R}^{n}\right) \otimes \mathcal{D}\left(\mathbf{R}^{m}\right) \quad$ algebraisches Tensorprodukt der

Testfunktionenräume $\mathcal{D}\left(\mathbf{R}^{\text {in }}\right)$ und $\mathcal{D}\left(\mathbf{R}^{m}\right)$

$\mathcal{D}\left(\mathbf{R}^{n}\right) \otimes_{\pi} \mathcal{D}\left(\mathbf{R}^{m}\right) \quad$ der Raum $\mathcal{D}\left(\mathbf{R}^{n}\right) \otimes \mathcal{D}\left(\mathbf{R}^{m}\right)$ versehen mit der projektiven Tensorprodukttopologie

$\mathcal{D}\left(\mathbf{R}^{n}\right) \tilde{\otimes}_{\pi} \mathcal{D}\left(\mathbf{R}^{m}\right) \quad$ Vervollständigung des Raumes $\mathcal{D}\left(\mathbf{R}^{n}\right) \otimes_{\pi} \mathcal{D}\left(\mathbf{R}^{m}\right)$

$u * v$

$T * u$

$T * S$

$\bar{\partial}$

$\mathcal{F}$

$\mathcal{F}^{\prime}$

$<\cdot, \cdot>$

$\|\cdot\|$

$l^{2}(\mathbf{K})$

$M^{\perp}$

$\operatorname{lin} M$

$[M]$

$\operatorname{dim} V$

$D(A)$

$\operatorname{Ker} A$

$\operatorname{Ran} A$

$\Gamma(A)$

$A^{*}$

$A_{F}$

$A+B$
Faltung (Faltungsprodukt) der Funktionen $u$ und $v$

Faltung der Distribution $T$ auf $\Omega$ mit der

Testfunktion $u \in \mathcal{D}(\Omega)=$ Regularisierte von $T$

Faltung der Distributionen $T$ und $S$, die die Trägerbedingung erfüllen

der Differentialoperator $\frac{1}{2}\left(\frac{\partial}{\partial x}+i \frac{\partial}{\partial y}\right)$ in $\mathcal{D}^{\prime}\left(\mathbf{R}^{2}\right)$

Fouriertransformation auf $L^{1}\left(\mathbf{R}^{n}\right)$ oder $\mathcal{S}\left(\mathbf{R}^{n}\right)$

Fouriertransformation auf $\mathcal{S}^{\prime}\left(\mathbf{R}^{n}\right)$

Skalarprodukt auf einem Vektorraum

Norm auf einem Vektorraum

Hilbert-Raum der quadratisch summierbaren Folgen von Elementen in $\mathbf{K}$

orthogonales Komplement einer Menge $M$ in einem (Prä-) Hilbert-Raum

der kleinste Teilraum, der die Menge $M$ enthält

der kleinste abgeschlossene Teilraum, der die Menge $M$ enthält $=$ Abschluß von lin $M$

Dimension des Vektorraumes $V$

Definitionsbereich des linearen Operators $A$

Kern oder Nullmenge des linearen Operators $A$

Wertebereich oder "Range" des linearen Operators $A$

Graph des linearen Operators $A$

der zu einem dicht definierten linearen Operator $A$ adjungierte Operator

Friedrichs-Erweiterung des dicht definierten positiven linearen Operators $A$

Formsumme der linearen Operatoren $A$ und $B$ 


\begin{tabular}{|c|c|}
\hline $\mathcal{L}(X, Y)$ & $\begin{array}{l}\text { Raum der stetigen linearen Operatoren } X \rightarrow Y \\
(X, Y \text { topologische Vektorräume über } \mathbf{K})\end{array}$ \\
\hline $\mathcal{B}(\mathcal{H})=\mathcal{L}(\mathcal{H}, \mathcal{H})$ & $\begin{array}{l}\text { Raum der beschränkten linearen Operatoren } \\
\text { auf dem Hilbert Raum } \mathcal{H}\end{array}$ \\
\hline $\mathcal{B}(\mathcal{H}, D)$ & $\{A \in \mathcal{B}(\mathcal{H}) \mid \operatorname{Ran} A \subseteq D\}$ \\
\hline$\hat{A}=(D, A)$ & $\begin{array}{l}\text { linearer Operator mit Definitionsbereich } D \text { und } \\
\text { Abbildungsvorschrift } A\end{array}$ \\
\hline $\mathcal{K}(\mathcal{H})$ & $\begin{array}{l}\text { Raum der kompakten Operatoren auf dem } \\
\text { Hilbert-Raum } \mathcal{H}\end{array}$ \\
\hline $\mathcal{P}(\mathcal{H})$ & $\begin{array}{l}\text { Raum der orthogonalen Projektionsoperatoren } \\
\text { auf dem Hilbert-Raum } \mathcal{H}\end{array}$ \\
\hline $\mathcal{S}(\mathcal{H})$ & $\begin{array}{l}\text { Raum der Spurklasse-Operatoren des } \\
\text { Hilbert-Raumes } \mathcal{H}\end{array}$ \\
\hline $\mathcal{U}(\mathcal{H})$ & $\begin{array}{l}\text { Raum der unitären Operatoren des } \\
\text { Hilbert-Raumes } \mathcal{H}\end{array}$ \\
\hline$\rho(A)$ & Resolventenmenge des linearen Operators $A$ \\
\hline$R_{A}(z)$ & $\begin{array}{l}\text { Resolvente des linearen Operators } A \text { im Punkte } \\
z \epsilon \rho(A)\end{array}$ \\
\hline$\sigma(A)$ & Spektrum des linearen Operators $A=\mathbf{C} \backslash \rho(A)$ \\
\hline$\sigma_{p}(A)$ & Punktspektrum von $A$ \\
\hline$\sigma_{c}(A)$ & kontinuierliches Spektrum von $A=\sigma(A) \backslash \sigma_{p}(A)$ \\
\hline$\sigma_{d}(A)$ & diskretes Spektrum von $A$ \\
\hline$\sigma_{a c}(A)$ & absolut stetiges Spektrum von $A$ \\
\hline$\sigma_{s c}(A)$ & singulär stetiges Spektrum von $A$ \\
\hline $\mathcal{H}_{p}(A)$ & unstetiger Teilraum von $A$ \\
\hline $\mathcal{H}_{c}(A)$ & stetiger Teilraum von $A$ \\
\hline $\mathcal{H}_{a c}(A)$ & $\begin{array}{l}\mathcal{H}_{c}(A) \cap \mathcal{H}_{s c}(A)^{\perp} \text { absolut stetiger Teilraum } \\
\text { des selbstadjungierten Operators } A\end{array}$ \\
\hline $\mathcal{H}_{s c}(A)$ & $\begin{array}{l}\text { singulär stetiger Teilraum des selbstadjungierten } \\
\text { Operators }\end{array}$ \\
\hline $\mathcal{H}_{s}(A)$ & $\begin{array}{l}\mathcal{H}_{p}(A) \otimes \mathcal{H}_{s c}(A) \text { singulärer Teilraum des } \\
\text { selbstadjungierten Operators } A\end{array}$ \\
\hline $\mathcal{M}_{b}(H)$ & $\begin{array}{l}\text { Unterraum der gebundenen Zustände des } \\
\text { selbstadjungierten Schrödinger Operators } H\end{array}$ \\
\hline $\mathcal{M}_{\infty}(H)$ & Unterraum der Streuzustände von $H$ \\
\hline $\operatorname{Proj}_{M}$ & $\begin{array}{l}\text { der orthogonale Projektor auf dem Unterraum } M \\
\text { eines Hilbert-Raumes } \mathcal{H}\end{array}$ \\
\hline
\end{tabular}

